\title{
Demagnetisation by Crossed Fields in Superconductors
}

\author{
Archie Campbell ${ }^{1}$, Mehdi Bagdhadi' ${ }^{2}$ Anup Patel ${ }^{3}$, Difan Zhou ${ }^{1}$, K.Y.Huang ${ }^{1}$, Tim Coombs ${ }^{1}$, and Shi \\ Yunhua \\ ${ }^{1}$ Dept. of Engineering, Trumpington St. Cambridge, ${ }^{2}$ University of Greenwich, ${ }^{3}$ Department of \\ Materials Science, Cambridge.
}

\begin{abstract}
A study has been made of the decay of the trapped magnetisation in superconductors when exposed to a crossed field. Numerical results have been compared with the theory of Brandt and Mikitik (4) which solves the problem for a thin strip superconductor. FlexPDE with the $A$ formulation and COMSOL with the $H$ formulation were both used. Simulations of a strip with a cross section aspect ratio of 20 showed good agreement with theory both for the case of a transverse field larger than the transverse penetration field and for one smaller. In the latter case the magnetisation saturates as predicted, however the simulations show a slow decay after many cycles. In the case of stacked YBCO tapes the movement of flux lines is very small and the effects of the reversible motion were investigated. This can decrease the decay initially for very thin decoupled tapes, but cause a steady decay after very large numbers of cycles. Simulations on stacked strips showed that the decay constant increased approximately linearly with the number of strips. When combined with the theory for one tape this can explain the very slow decay observed in previous experiments.

Experimental results were qualitatively in agreement with theory and simulations but showed some discrepancies. However there are a number of differences between the experimental situation and theory so good agreement is not expected.
\end{abstract}

\section{Introduction}

In most applications of bulk superconductors using trapped fields the bulk will be exposed to external fields which will change in both angle and magnitude. While changes in in magnitude are easily incorporated in the Bean model, changes in angle lead to much more complex situations, in many cases involving flux cutting and force free configurations. However many experimental measurements have shown that in general trapped fields are always reduced by the application of crossed fields and this effect may limit the application of bulk superconductors in electrical machines. Most of the experiments were at relatively high fields compared with the crossed fields and directed at elucidating the physics, rather than practical applications of bulk superconductors in motors and generators. In this paper we consider geometries in which the induced currents are mostly perpendicular to the fields so that the standard Bean model can be applied.

One of the first observations was that of Sychev et. al. (1), who applied an oscillating field to a wire carrying a current and observed a DC voltage. Sakamoto et. al. (2) used a thin film carrying a current in an applied field and measured the decay of the trapped magnetisation when a transverse field was applied, as in this paper. They showed that the flux lines pivoted about two points alternately and 'walked' out of the film, a bit like penguins heading for their breeding grounds in the Antarctic. They proposed an approximate theory which assumed a uniform current density in the film. The DC voltage generated by an AC field was termed the 'dynamic resistance' and the clearest explanation of this in in reference (3), albeit, for a rather different geometry, i.e. a field parallel to the trapped field in the solenoid. This is described in more detail in $\S 2$. A complete theory for a thin strip was published by Brandt and Mikitic (4), and this will be compared with modelling and experiments below.

The discovery of high $\mathrm{T}_{\mathrm{c}}$, superconductors, and their processing into bulk pellets, made experiments much easier. Vanderbemden et. al. (5) measured the collapse of the trapped magnetisation where a cross field comparable to the trapped field was applied to an YBCO bulk. It was found that the magnetisation was 
greatly reduced within a few cycles. The results were compared with a numerical 2D model and were in qualitative agreement. However the decay was a factor of five slower than predicted by simulations. Experiments on stacked YBCO tapes showed a remarkably low decay rate, even in cross fields many times larger than the parallel field required to penetrate the YBCO layer $(6,7)$. On the other hand Celebi et. al. performed similar experiments and modelling on stacks of BSCCO tapes and found agreement between the decay and the modelling (8). The experimental situation is therefore somewhat confused. The most relevant theory is that of and Brandt and Mikitik (4), although it was developed for a rather different context. In their paper they showed how shaking flux lines could reveal the reversible magnetisation by reducing the trapped magnetisation to zero. However it makes clear the physics of the process and the most relevant parameters for the purposes of predicting the decay of a trapped magnetisation. The most important are the aspect ratio and the ratio of the transverse penetration field to the applied transverse field.

\section{The Dynamic Resistance and Current Distribution}

\subsection{The Bean model in slab geometry.}

The principle as described in (2) is illustrated in fig.1. This shows the Bean model distribution in a slab carrying a current less than critical, which then has a parallel field $\mathrm{B}_{\mathrm{o}}$ which is larger than the self field, applied to it. The field distribution is in red in fig. 1a.

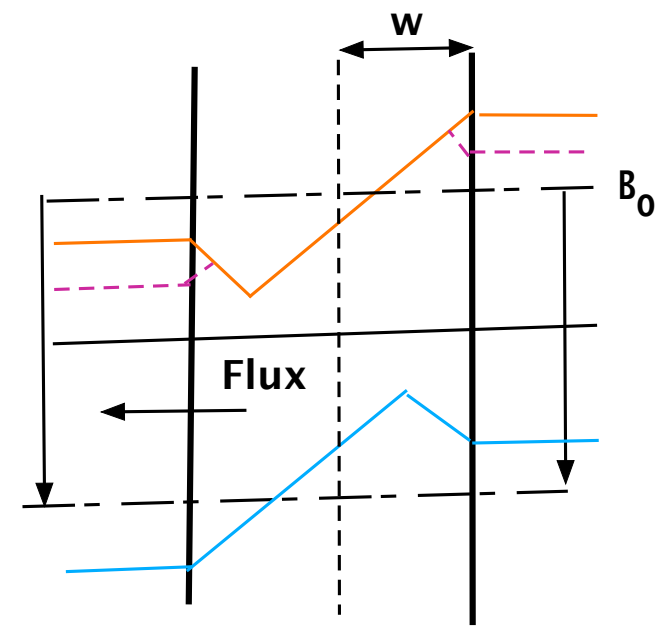

Fig.1a First a current, then $B_{o}$, then $-B_{o}$ are applied.

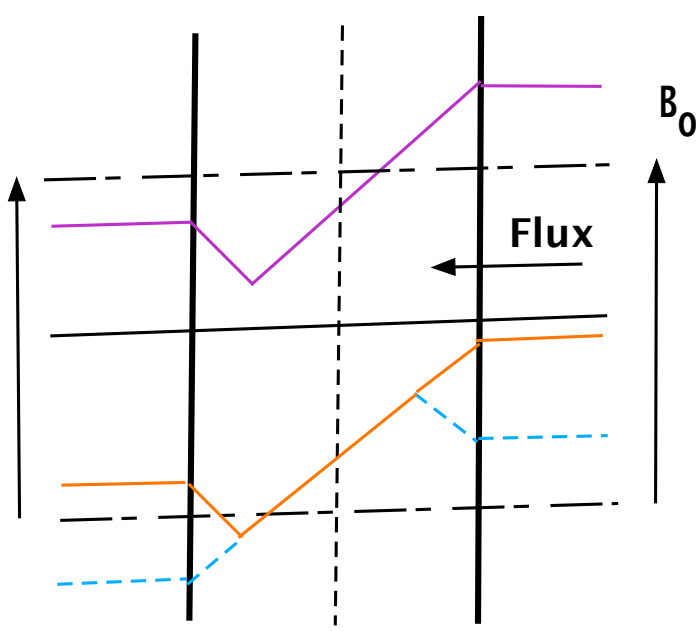

b) The field $-B_{o}$ is raised to $B_{o}$.

Now suppose we oscillate the applied field by a small amount along the purple dotted lines in fig.1a. The field penetrates from each side, but does not reach the point where $J$ changes sign, the cusp of the field distribution. In this case the loss is just the same as if there were no current flowing.

However if the amplitude is now increased until the two critical states on the left side merge there is a sudden change in the loss. As $B_{o}$ is further reduced all the flux up to the right hand cusp moves out of the sample to the left, and the field distribution follows the lower blue curve. The cusp is a position where $\boldsymbol{E}=0$ and no flux crosses it. It is a distance $x$ from the centre where $x=w I / I_{c}$.

If we now bring the applied field up to its original value the reverse process takes place. When the critical states on the right merge the field distribution in red moves up until we reach the starting point in fig.1a. Flux moves in from the right to fill the material up to the cusp on the left.

The result is that on each cycle flux is effectively pumped from right to left creating a DC component to the electric field, which is termed the 'dynamic resistance'. The electric centre, where $\boldsymbol{E}=0$, switches discontinuously between the two cusps of the critical state on each cycle and the flux is transferred from right to left on both up and down parts of the cycle. 
The threshold amplitude at which the change takes place is the current dependent penetration field. It is easily calculated from the Bean model and varies linearly from zero at the critical current to the usual penetration field $B_{p}=\mu_{o} j_{c} w$ where $w$ is the half width and $j_{c}$ the critical current density. The current dependent penetration field is $B_{p}(I)=B_{p}(1-I / I c)$. In this geometry it can be applied to a slab carrying a constant current, or approximately to a solenoid carrying a persistent current. Note that the penetration of the currents due to the applied field is $w\left(1-I / I_{c}\right)$ and is independent of the amplitude of the oscillating field. Penetration is small if the current is close to critical, even if the field applied is much greater than the penetration field. The magnetic field however penetrates right into the sample. This apparent contradiction is due to the fact that the current plus a small part of the oscillating field current saturates the sample with the critical current so that further changes in field can penetrate the whole sample. This effect is important in the strip geometry used in this paper and discussed below.

In a solenoid the results are that, since a trapped field implies the wire is carrying its critical current, initially the current dependent penetration field is zero and flux is transferred out on every cycle. However this reduces the field in the solenoid, and hence also the current, so that at some point the field drops to the current dependent penetration field and no further decay takes place. However if the amplitude is greater than $B_{p}=\mu_{o} j_{c} w$ the solenoid field decays to zero.

Brandt and Mikitik went to the opposite geometry (4) and considered a long very thin strip of superconductor with a perpendicular trapped field exposed to a ripple field in the $y z$ plane as in figs.2-4. This is the only geometry which can be entirely explained by the Bean model since all currents are perpendicular to the field, except at the ends.

\section{The Brandt and Mikitic Theory}

In ref(4) Brandt and Mikitic show how the shaking of flux lines by a crossed field leads to the decay of a trapped magnetisation. This is for the case of a long thin strip with the trapped field normal to the strip, so is exactly what is needed to explain the decay of the trapped field in superconducting tapes. One difference is that they assume a large constant applied field normal to the tape. This allows an attractive interpretation in terms of flux lines 'walking' out of the sample, a picture first suggested by Sakomoto et. al. (2).

The result is independent of this applied normal field so can be applied to the trapped field in tapes where there is no other field in this direction. This is to be expected since any solution based on the Bean model with a constant $j_{c}$ is independent of any applied constant field.

\subsection{Current Distributions}

Figure 2 shows the currents in a strip, long in the $x$ direction, with a trapped field in the $z$ direction. Figure 3 shows the currents if a field parallel to the surface is applied, with no trapped field. The applied field is greater than the penetration field in this direction.

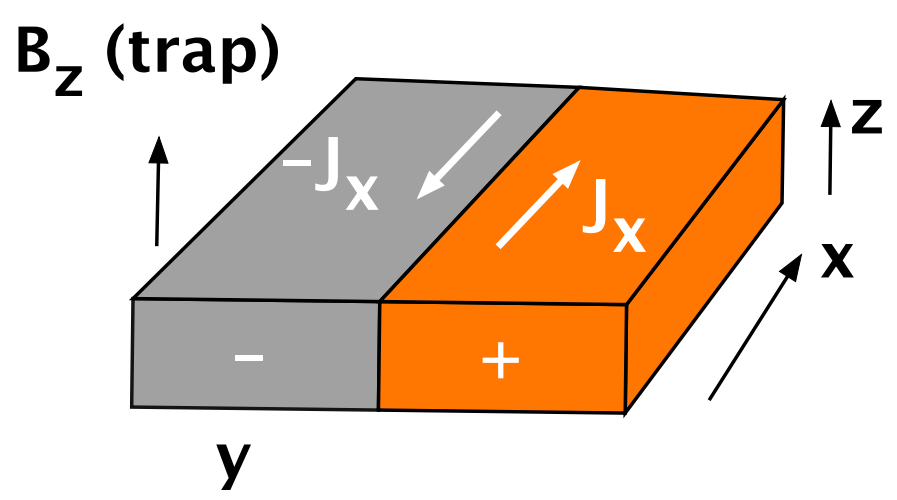

Fig 2. Currents for a trapped $B_{z}$

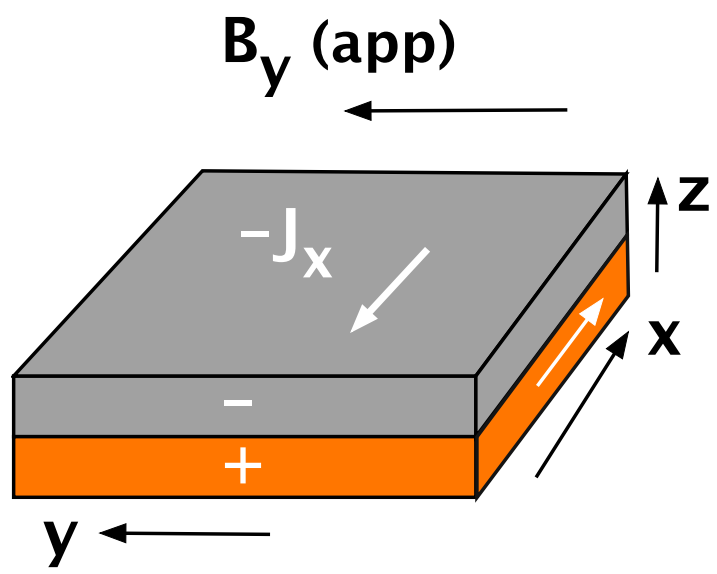

Fig.3 . Currents for a parallel field. 
Figure 3 shows the currents when a cross field is applied to a sample with a trapped field. Where the induced currents are parallel to the trapped currents there is no change, since the currents are already critical.

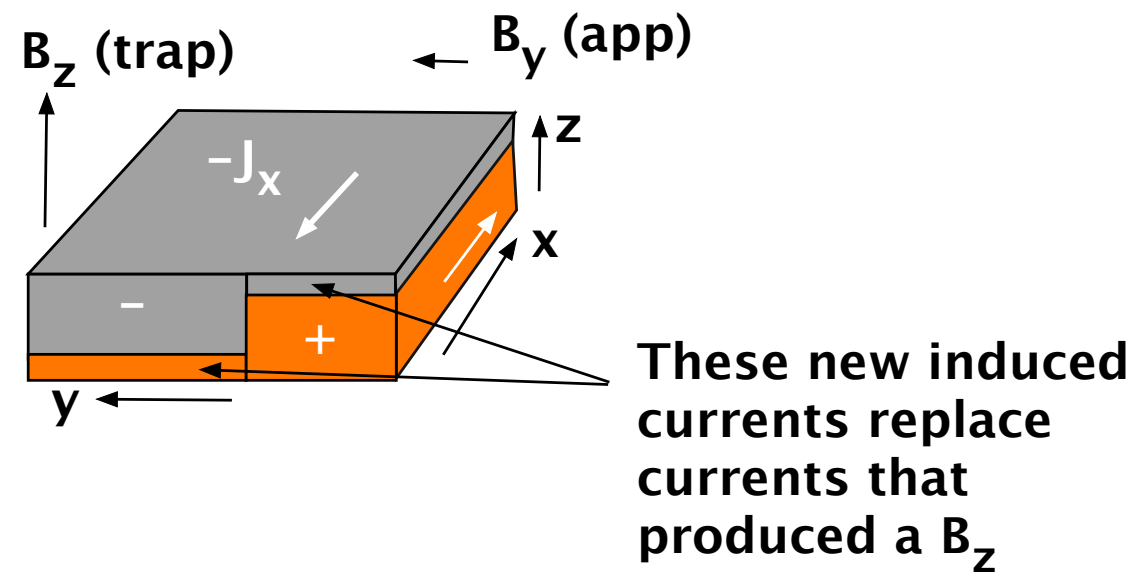

Fig. 3. A trapped field with an applied cross field.

Where the induced currents are antiparallel to the trapped currents, currents in the opposite direction are induced. Although the applied field is greater than the penetration field these do not penetrate to the centre, but to a much smaller distance. This is partly because reversing these currents requires twice the field to generate them. However the penetration distance is much smaller than even that implies. This can be seen from fig.1, which is for a different geometry, but the same argument applies to the generation of the critical state. The cusp where the current reverses is at a distance $w I / I c$, from the centre, so the penetration of the oscillating field is $w\left(1-I / I_{c}\right)$ where $I$ is the current generating the trapped field. Once the cross field exceeds the current dependent penetration field, which is when it penetrates this distance, the sample is saturated and further increase in field increases the field everywhere uniformly, so there is no change in the current distribution.

\subsection{The Vortex Picture.}

Brandt and Mikitik assumed a thin strip in the $x y$ plane and a large applied field in the $z$ direction, the direction of the trapped field. This leads to the 'walking' motion described in (2). The process is illustrated in fig.5. At the cusps of the current distribution in fg. 1 there is no electric field so the flux does not move. This position changes discontinuously on each cycle. For the strip geometry these points are pivot points about which flux lines rotate. As the pivot point switches from one side to the other the flus lines 'walk' out of the sample. For the trapped field situation described in this paper there is no imposed large external field so this picture does not work, but the conclusions are the same.

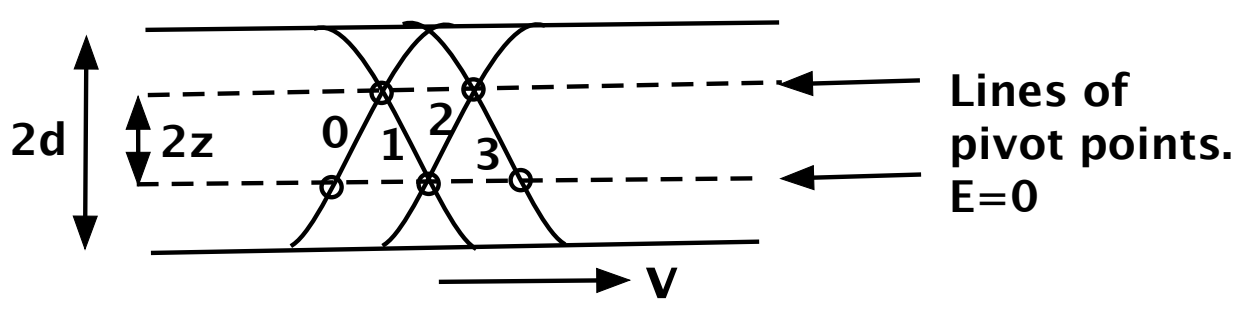

Fig. 5. A flux line follows $0,1,2,3$ and moves to the right. 
We define $J(x)$ as the sheet current, i,e the current integrated across the superconductor at a point $x$. $J_{c}$ is the maximum sheet current and $J_{c}=2 j_{c} d$ where $d$ is the half width. The transverse penetration field is $\mathrm{B}_{\mathrm{p}}=\mu_{o} j_{c} d=\mu_{o} J_{c} / 2$ and the applied transverse field is $B_{T}$.

The distance moved per cycle is $\Delta x=\frac{2 J}{j_{c} B_{z}}\left[B_{T}-B_{p}(J)\right]$ and the mean velocity gives the DC component of the electric field, which reduces the trapped current and field. The current dependent penetration field is $B_{p}(J, x)=\mu_{o}\left(J_{c}-|J(x)| / 2\right)$. The trapped moment is $M_{o}=-w^{2} J_{c}$.

Up to this point there is a close parallel between the expressions for the current distributions for the cases of field parallel and normal to the strip. The difference arises in calculating the effect of the electric field over a cycle on the change in current. In the case of fig. 1 it is relatively easy to relate the decrease in current to the flux transferred, but this is more difficult in the thin strip geometry and requires a numerical solution to an integral equation. The results are shown in fig. 2 of ref. 4 and can be summarised as follows.

Transverse fields much lower than the trapped field are unlikely to be of much practical importance but for fields comparable to, but less than, the penetration field $B_{p}$, the magnetisation drops within a number of cycles, which is about $w / 4 d$, to a value $M_{s}=M_{o}\left(1-B_{T} / B_{p}\right)$. For transverse fields larger than the penetration field $B_{p}$ the magnetisation decays exponentially with a characteristic number of cycles $n_{o}$ where

$$
n_{o}=\frac{w B_{p}}{1.28 \pi d\left(B_{T}-B_{p}\right)}
$$

It can be see that for thin tapes with $B_{p}<<B_{T}$ the decay constant depends on the product of the ratio of $B_{p}$ to $B_{T}$ multiplied by the aspect ratio. In other words, although in general for a typical puck a transverse field greater than the penetration field in the transverse direction will cause a collapse of the magnetisation within a few cycles, if the sample is very thin, as in a tape, the decay is much slower. The theory shows it is not possible to model the decay using a thicker film by lowering the current density in proportion. The aspect ratio and ratio of $B_{T}$ to $B_{p}$ are two independent parameters. Another important result is that the decay time does not depend on the large applied $z$ field which was used in the derivation, and to describe the walking motion. We can therefore apply the theory to a trapped magnetisation with no applied field. There should be no frequency effect, although one was observed experimentally in ref (6), even at low frequencies.

\section{Comparison with FE analysis. \\ 4.1 Large Transverse Field}

A comparison between the Brandt Mikitik theory and FE modelling was first made with long strip with a rectangular cross section. The width was $10 \mathrm{~mm}$ and the half thickness $0.5 \mathrm{~mm}$. This used FlexPDE and the $A$ formulation. This method uses a parameter $A_{o}$ which is related to the distance the flux lines can move reversibly. It is described in detail in ref. (6). It uses an average force-displacement curve for the interaction between vortices and the pinning centres. This consists of an initial linear section up to a certain displacement, beyond which the force saturates at $\mathrm{BJ}_{\mathrm{c}}$. Since the displacement of the flux lines is proportional to the vector potential the balance between the Lorentz force and the vector potential leads to the partial differential equation $\operatorname{curlcurl}(A)=\mu_{\mathrm{o}} j_{c}\left(1-\exp \left(-A / A_{o}\right)\right)$. The solution to this gives the critical state directly without having to invoke a time dependence, or V-I characteristic. The ideal critical state is reached in the limit of $A_{o}$ tending to zero. For the critical state regime the addition of a uniform constant field makes no difference to the equations, so the results are inevitably independent of the applied field except in so far as it affects $j_{c}$. This is in agreement with the theory. This sample with an aspect ratio of 20 was thin enough for the theory to apply, but not so thin that it caused difficulties in convergence to a solution. 
Figure 6 shows the decay of the relative magnetisation $M / M_{o}$ for a cross field of $300 \mathrm{mT}$. The critical current density assumed was $10^{4} \mathrm{~A} / \mathrm{cm}^{2}$ so the penetration field $B_{p}=63 \mathrm{mT}$. $B_{T} / B_{p}=4.8$. The magnetisation is normalised, and the graph of $B_{T}$ is shown in arbitrary units for illustration. The theoretical characteristic decay constant is 0.66 cycles. The agreement between theory and experiment is excellent. The trapped magnetisation vanishes within three cycles

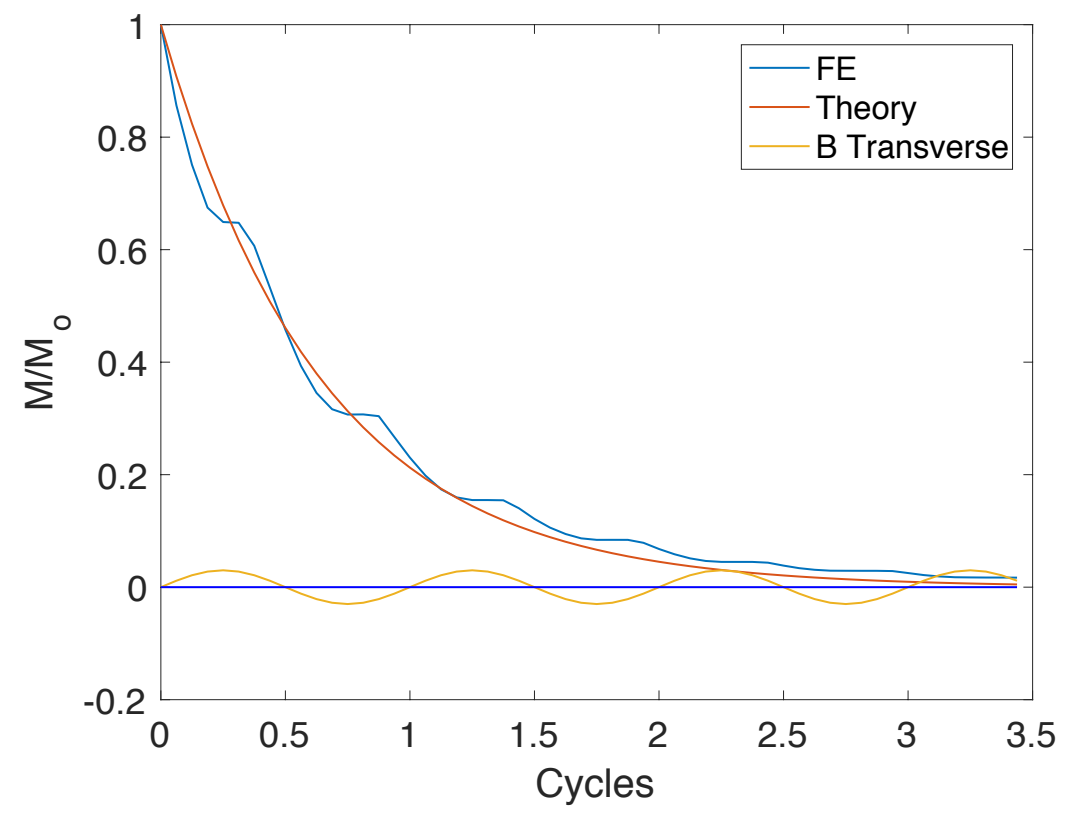

Fig.6. Ratio of magnetisation $M$ to initial value $M_{o}$ for $B_{T}=4.7 B_{p}$.

Figure $7 \mathrm{a}$ shows the current distribution at the starting point, at a cross field of $300 \mathrm{mT}, 7 \mathrm{~b}$ at $-300 \mathrm{mT}$ and $7 \mathrm{c}$ after six cycles. Red is plus $j_{c}$ and purple minus $j_{c}$., The $y$ axis is expanded to equal the $x$ axis for clarity. Fig.7e shows the true geometry. It can be seen the currents correspond qualitatively to those in figs. 2-4, but the sheet current varies along the sheet as predicted by the theory.

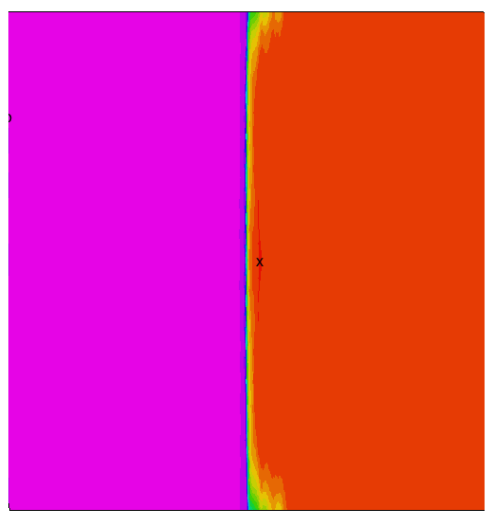

Fig.7. Current distributions. a) Start

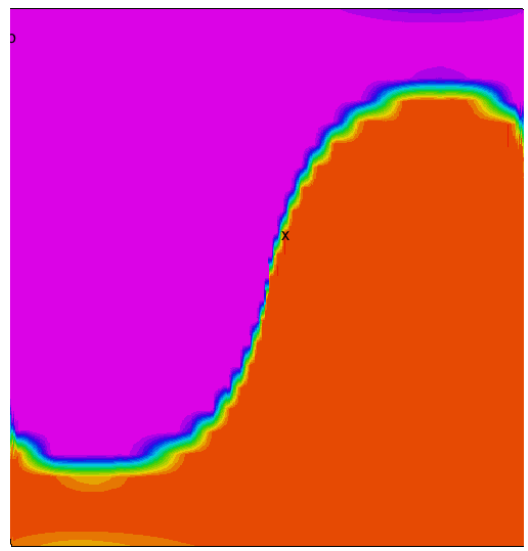

b) Apply cross field of $300 \mathrm{mT}$ 


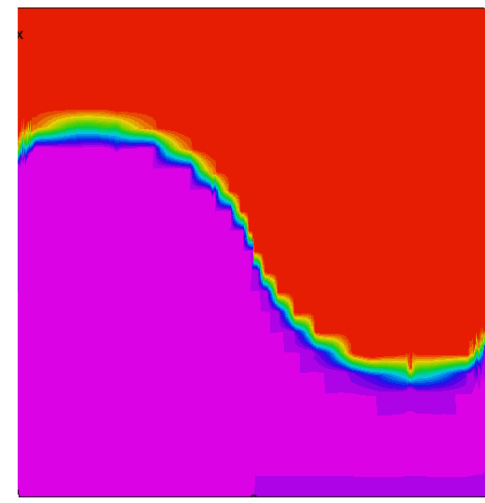

c) Apply $-300 \mathrm{mT}$

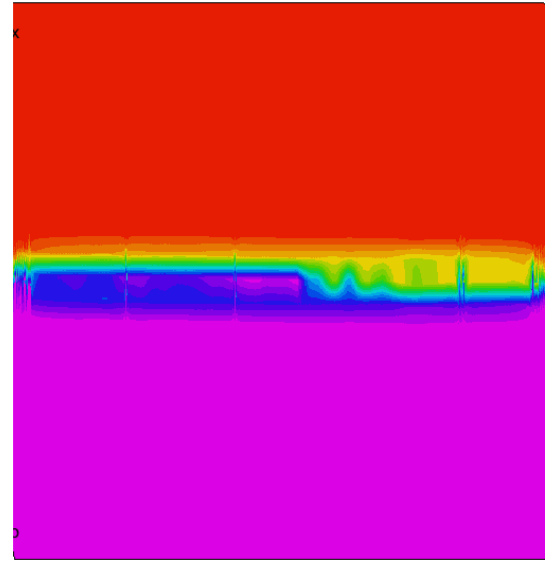

d) After six cycles

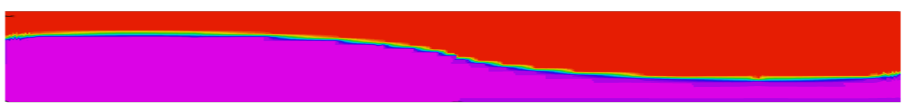

e) The actual geometry at $-300 \mathrm{mT}$

Figure 8 shows the flux lines at the start and at $300 \mathrm{mT}$ cross field. It can be seen that because there is no applied normal field the 'walking flux line' picture does not work, but the results nevertheless agree with the theory.

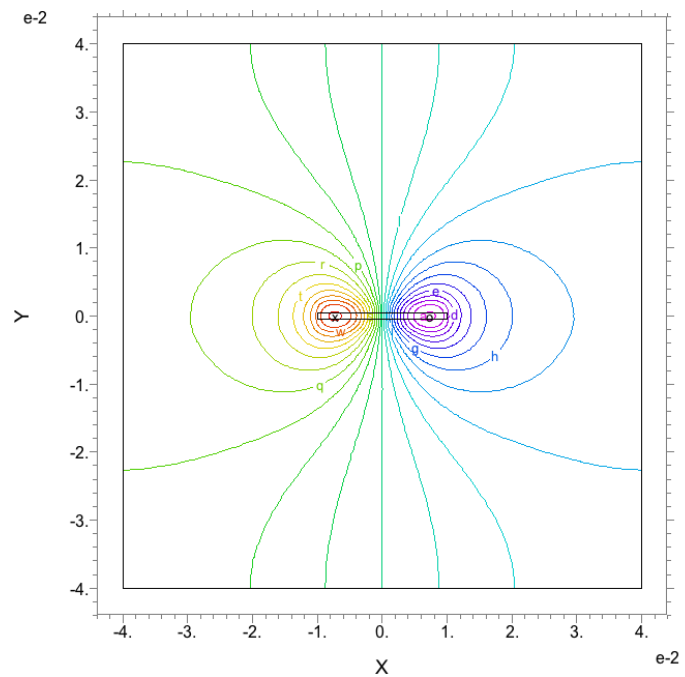

Fig.8. a) Starting trapped lux lines

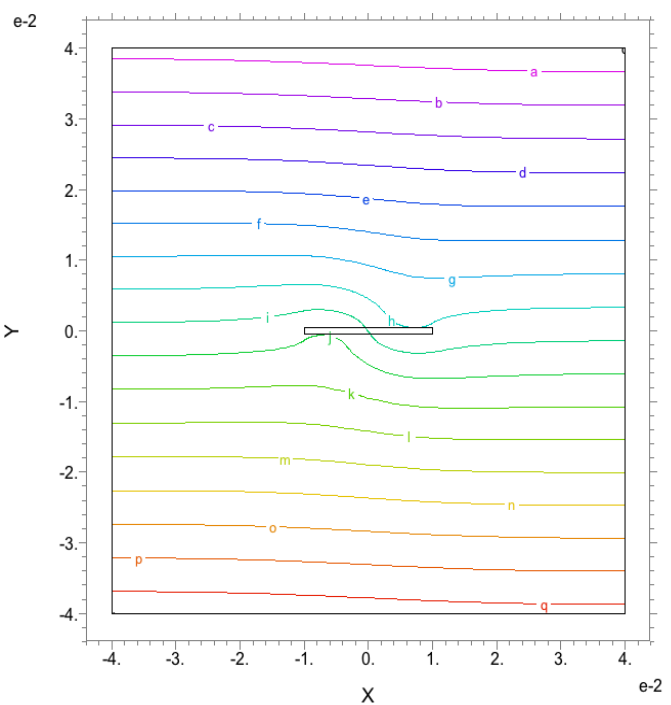

b) Flux lines at $300 \mathrm{mT}$ cross field

\subsection{Small Transverse Field}

In figure 9a comparison is made for a lower transverse field $B_{T}=0.8 \mathrm{~B}_{\mathrm{p}}$. Again good agreement is seen up to 30 cycles. The simulated magnetisation decays to the predicted value of $M_{o}\left(1-B_{T} / B_{p}\right)=0.2 M_{o}$. 


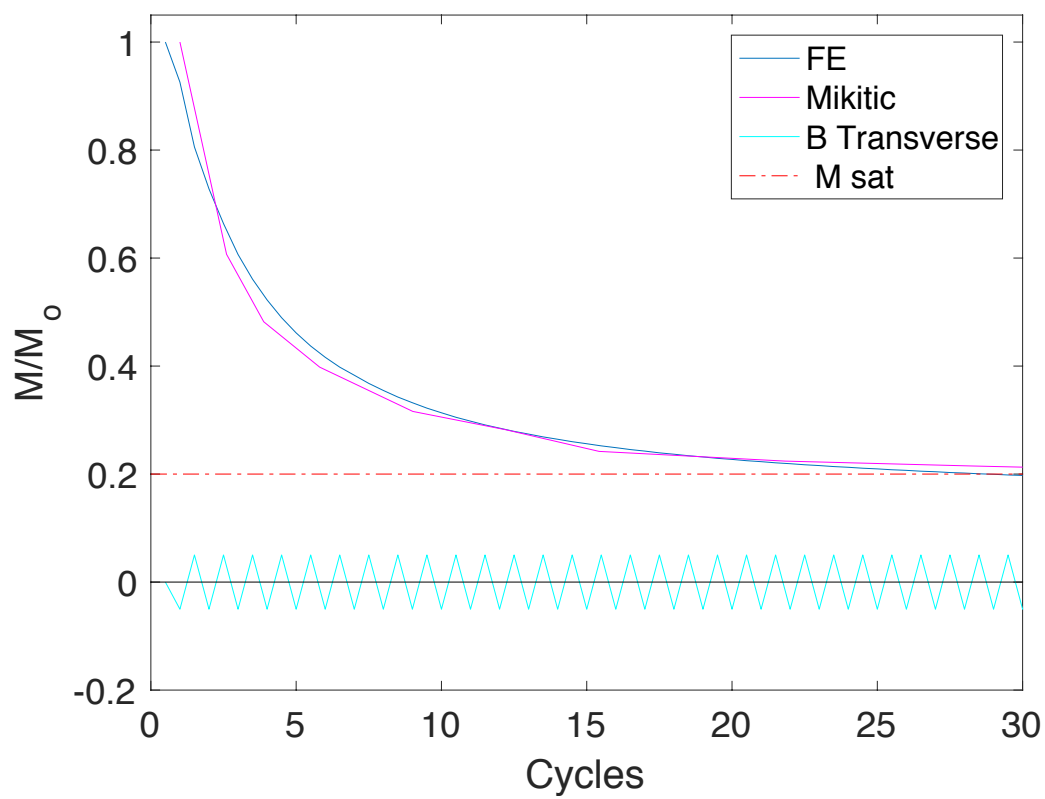

Figure 9. Magnetisation decay for lower transverse field $\left(0.8 B_{p}=10 \mathrm{mT}\right)$

However for larger numbers of cycles the magnetisation did not stay constant but decayed slowly. This is shown in fig. 10 where 250 cycles are plotted.

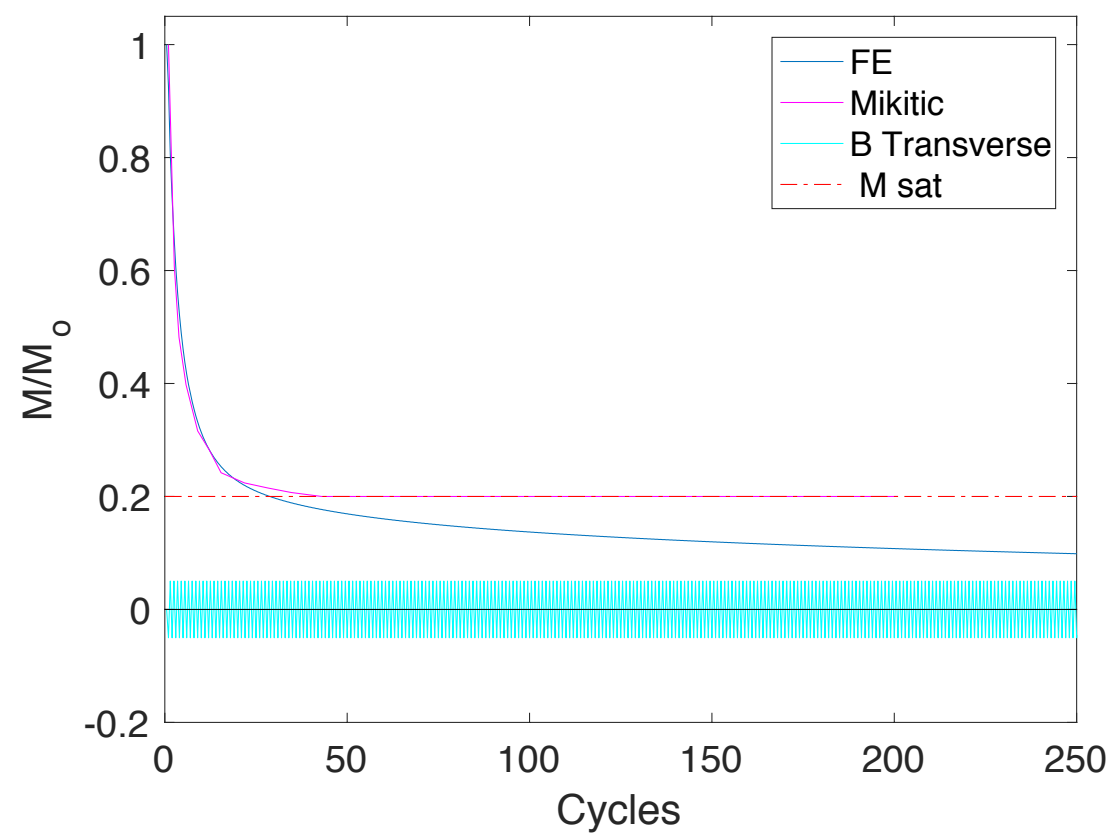

Fig.10. Magnetisation decay for $B_{T}=10 \mathrm{mT}$ and 250 cycles. 
Figure 11 shows the current distributions.

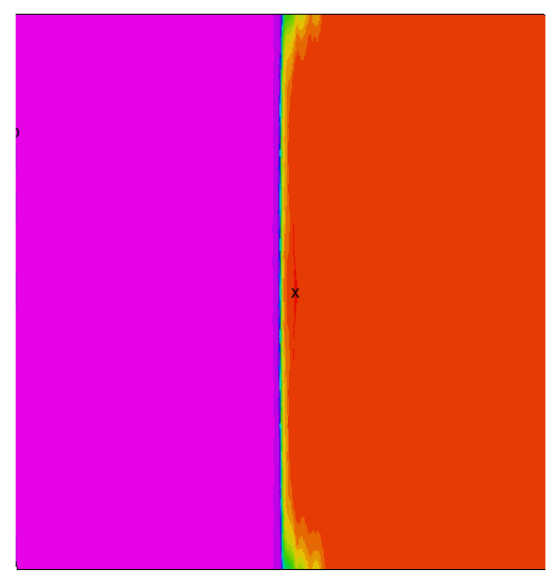

Fig11. a) Starting current distribution

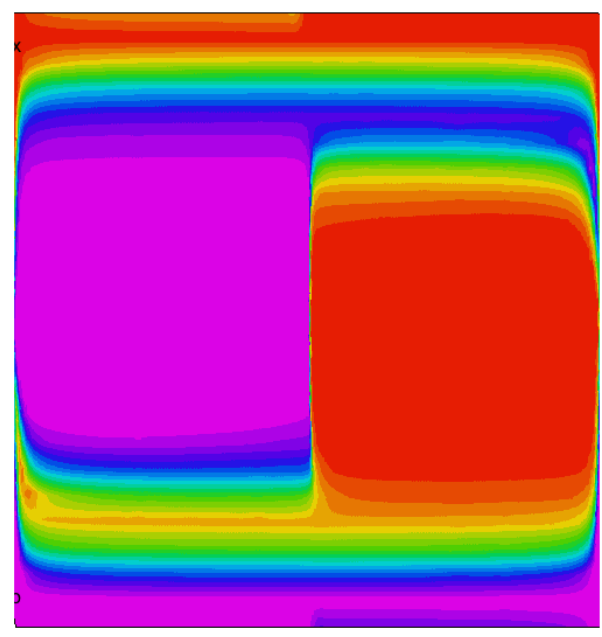

c) After30 cycles

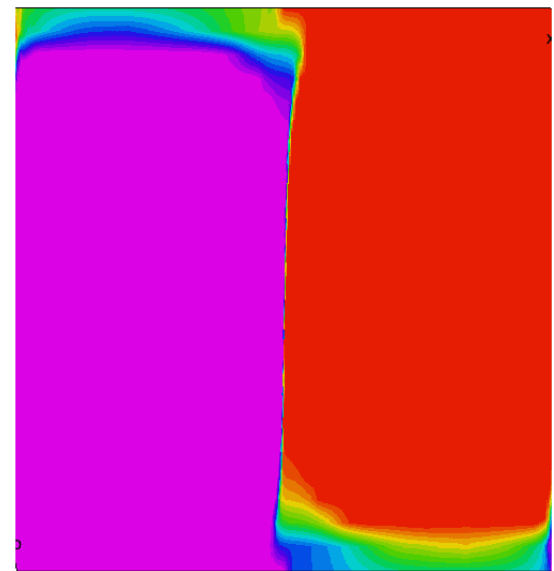

b) $10 \mathrm{mT}$ cross field

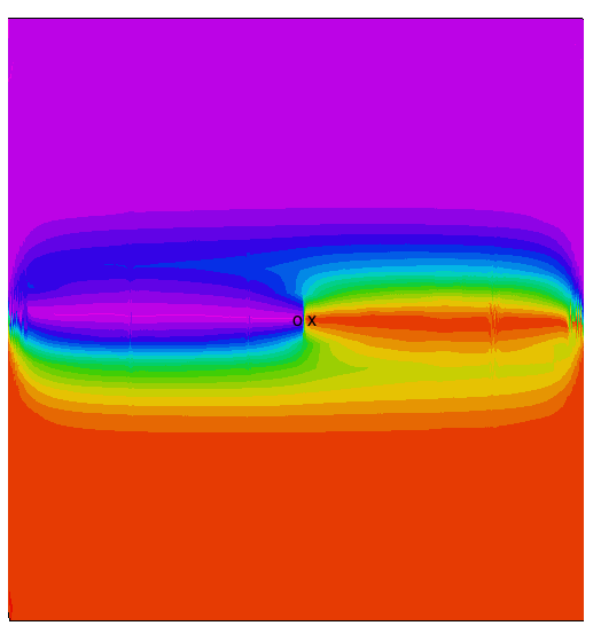

d) After 200 cycles

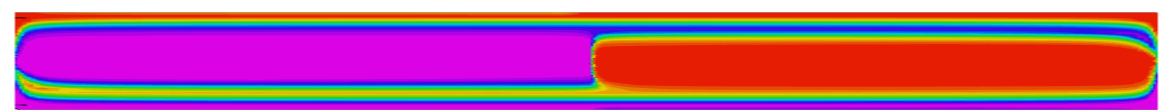

e) The actual geometry after 30 cycles

The reason for this decay is not clear but one possibility is that it is connected with the value of the parameter $A_{o}$. There are two effects. The first is that if the flux lines are not moving far they do not become unpinned so that they just oscillate within their potential wells. In this case there is no decay of the trapped moment. An extreme case would be if the superconducting atomic layers were decoupled from their neighbours. There is no way that a critical state can be built up within a single atomic layer so a field trapped in such a layer would not decay. A similar but lesser effect will occur in layers of thickness comparable to the penetration depth such as in an YBCO tape. This could cause a slower decay in very thin layers than predicted by the Brandt-Mikitik theory. 


\subsection{The effect of reversible displacements}

Figure 12 compares the solution with $A_{o}$ at a value small enough to give a good approximation to the critical state with one ten times larger. It can be seen that at low numbers of cycles the large $A_{o}$ gives a larger decay constant.

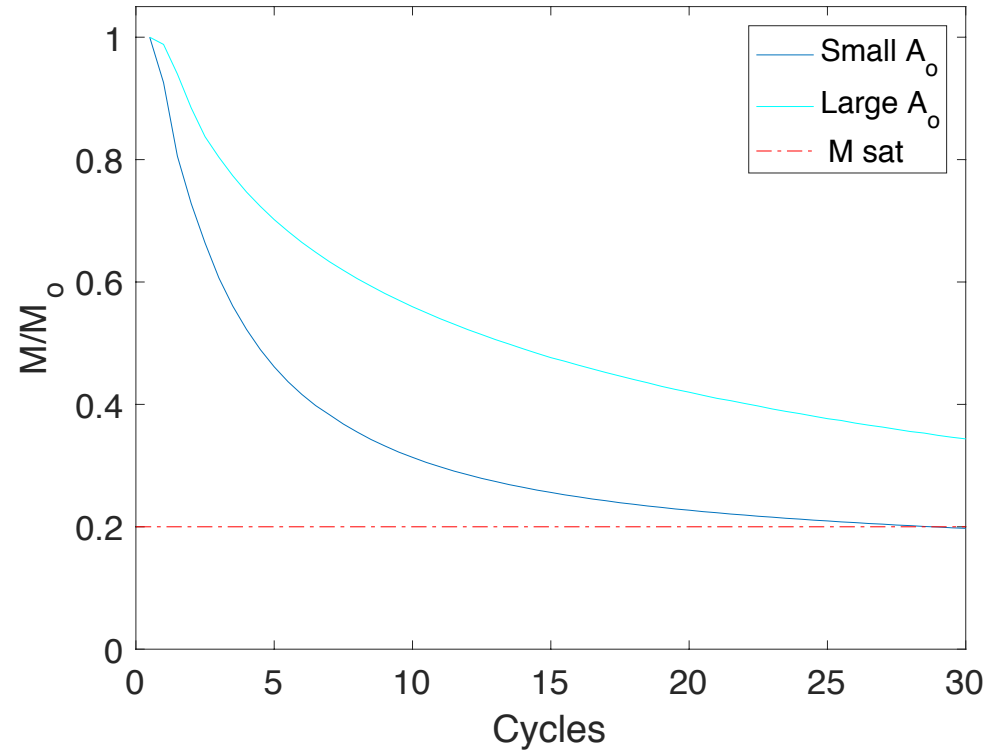

Fig.12. The decay of magnetisation for a small value of $A_{o}$ (critical state) and one ten times larger

There is however an opposite effect occurring at large numbers of cycles, when the amplitude of the vortex oscillation is insufficient to create the critical state. Figure 13 shows the effect of a larger $A_{o}$ on the decay of magnetisation after 250 cycles and it can be seen that the larger $A_{o}$ causes a larger decay.

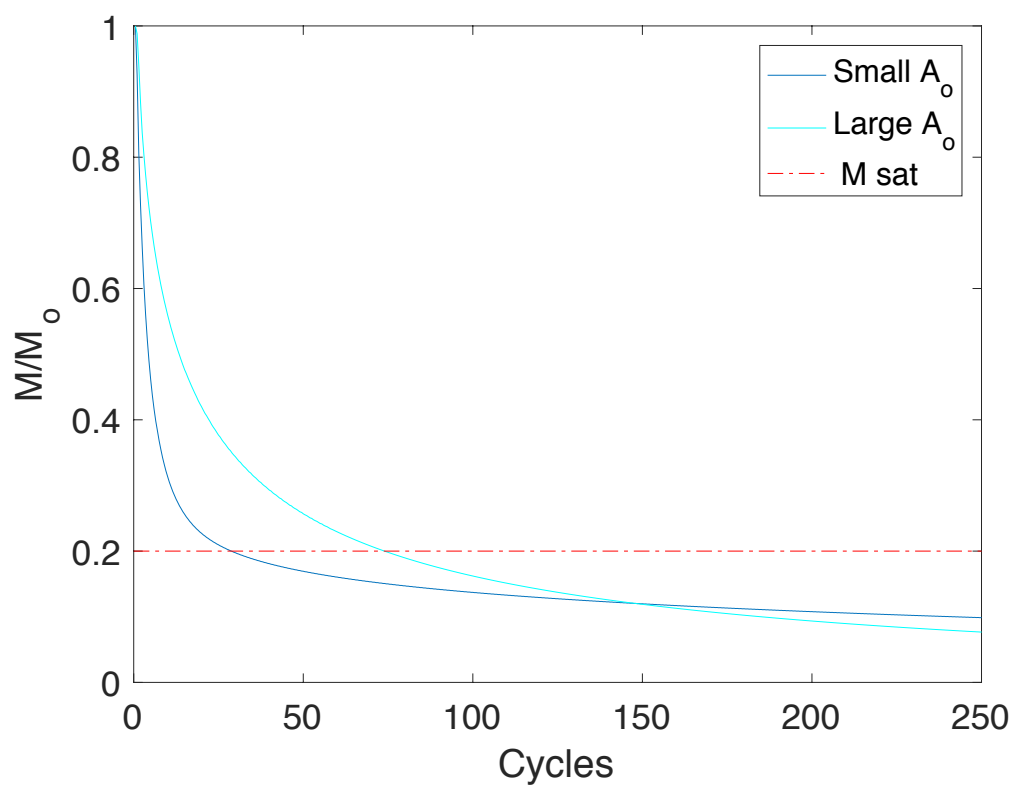

Fig.13. After many cycles the decay is more for large $A_{0}$. 
This may be explained by finding the minor loops of the vortex oscillation which do not reach the critical state. Figure 14 shows the force-displacement curve with a minor loop after the critical state has been built up. We do not know the shape of such a minor loop in detail but we assume that the initial slope on any reversal is the same as the initial slope of the virgin state. (This is interesting in itself, but seems to be true experimentally). Also for large reverse displacements the curve must tend to minus one. Using an exponential to bridge the gap gives the loops in fig 14. The loops do not close, and in fact it is very difficult to think of any model in which the curves form closed loops if they are to approach plus and minus one for large displacements. It would require a discontinuity where the curve meets the critical state. The result is that the critical state decays until the current is zero. Only at this point are the minor hysteresis loops symmetrical and close. A similar effect occurs when levitated magnets are oscillated, but in most cases this is a larger scale phenomenon and can be explained in terms of the critical state model. This shaking process is common to all hysteretic systems including permanent magnets and plastic deformation. It is also used by mechanical engineers to eliminate frictional effects. Another example of this general result is the decay of the trapped field in a solenoid illustrated in fig.1. The effects are however normally not very great and it remains to be seen if they are relevant to shaking in superconductors. The best measurements of the reversible limit in YBCO are in ref. (10) which found distances of only a few nanometres. This seems a bit small to explain the effects.

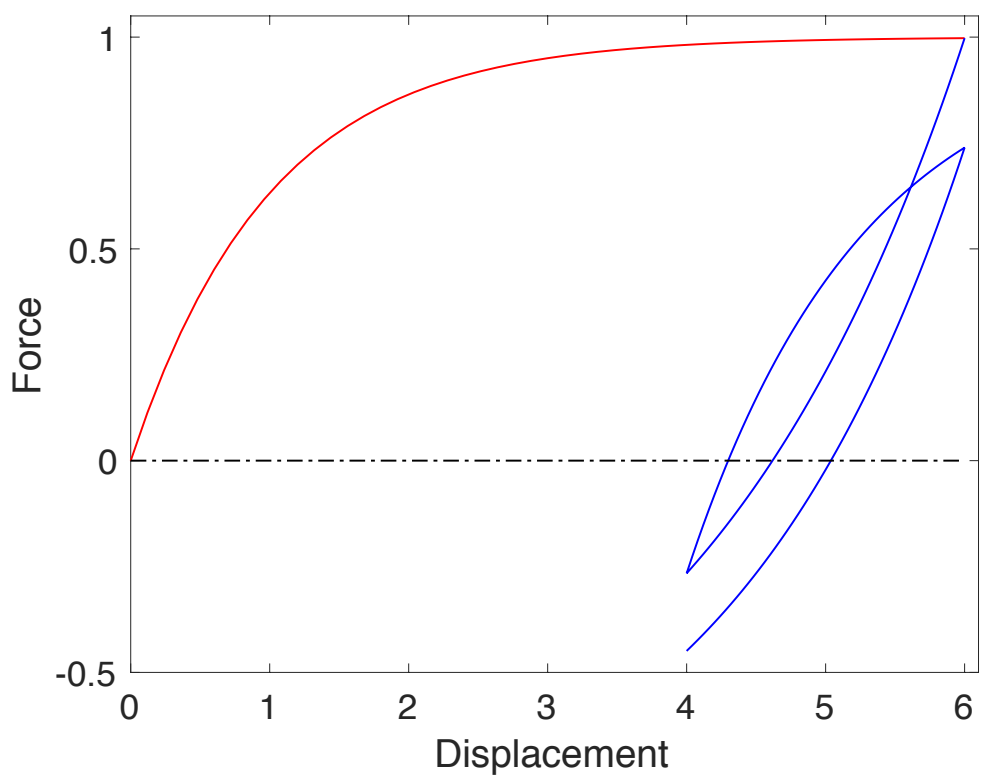

Fig.14 Minor loops do not close until the magnetisation is reduced to zero.

\section{Experimental Results}

\subsection{Bulk Samples}

A number of experimental results were compared with the theory and simulations. It must be recognised that the experimental situation is rather different from the theory. This is a 2D theory applied to a long strip while most experimental samples were roughly square in the $x y$ plane. Also there were none available of the aspect ratio in the simulations above. Another difference is that what was measured is the peak trapped field rather than the magnetisation, which is more easily calculated in the theory. The peak field is available from the simulations, but has a sharp peak near the centre so it is easy to get an inaccurate value experimentally.

The material is assumed isotropic, which can affect short samples, and also $j_{c}$ is assumed constant and uniform whereas in a one micron YBCO film a large part of the current is probably carried on the 
surfaces. For all these reasons we can only expect qualitative agreement, but it is still useful to see to what extent existing experiments agree with the theory in order to guide a more directed program.

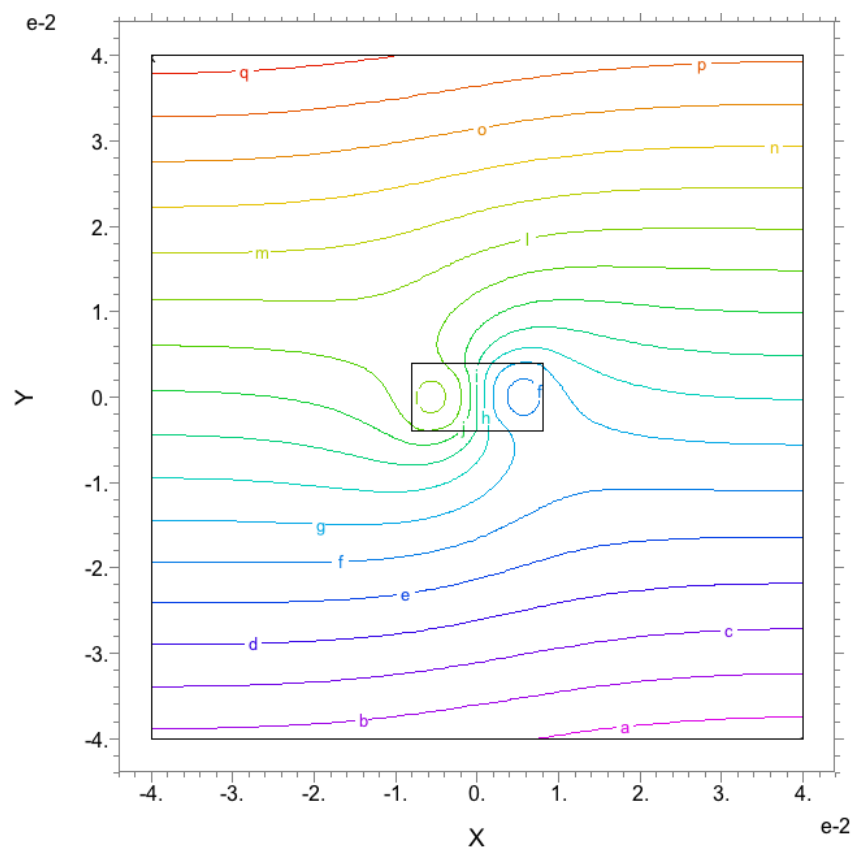

Fig.15 Flux lines in a puck.

Figure 15 shows the cross section of a puck of diameter $16 \mathrm{~mm}$ and total thickness $8 \mathrm{~mm}$. for which the decay was measured.

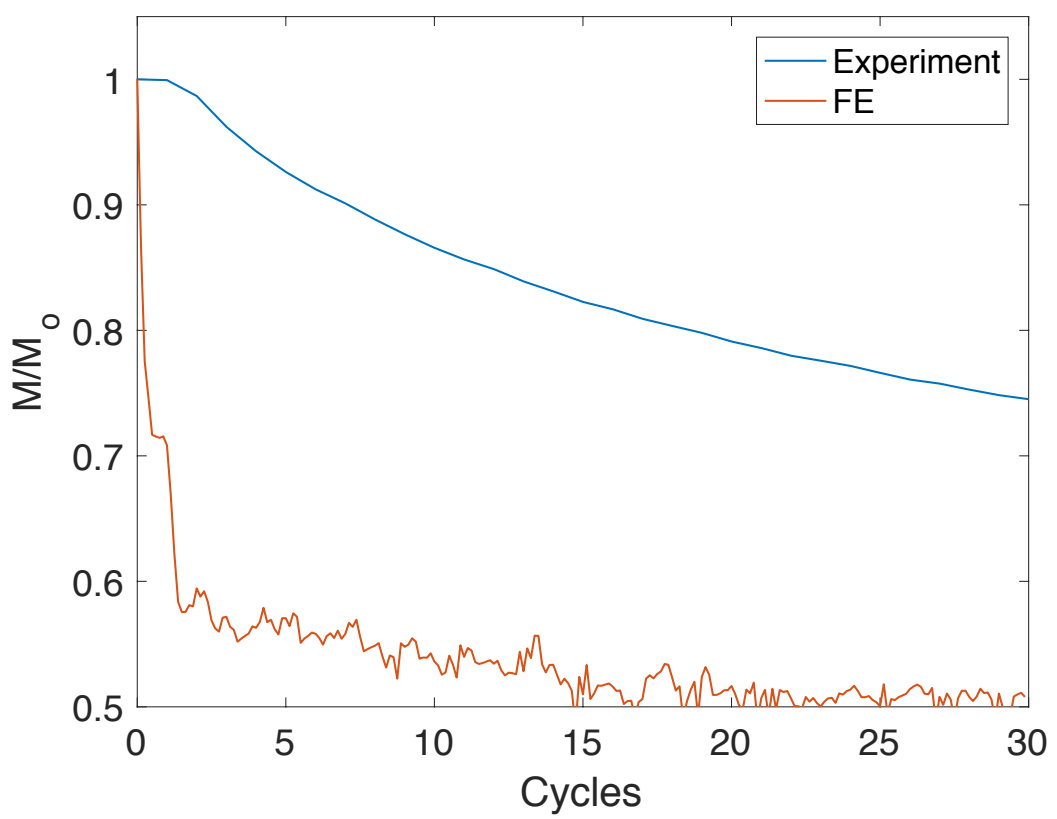

Fig.16 The decay of the central trapped field in the puck. 
A simulation was done assuming an infinite strip with the same cross section. The critical current density was calculated from the peak surface trapped field and the penetration field derived. In this case $B_{p}=600 \mathrm{mT}$. The transverse field was $B_{T}=120 \mathrm{mT}$. the ratio is 0.17 so the theory predicts a relative saturated magnetisation of 0.83 . The simulation shows a sharp drop over the first few cycles and saturation at about 0.5 , although it continues to decrease. The experiments show a continuous and much slower decrease. Since the aspect ratio is 0.5 we do not expect the Brandt-Mikitic theory to be accurate, but with an aspect ratio of 2 we expect a collapse to the saturation value within a few cycles as observed.

\subsection{A Single Tape}

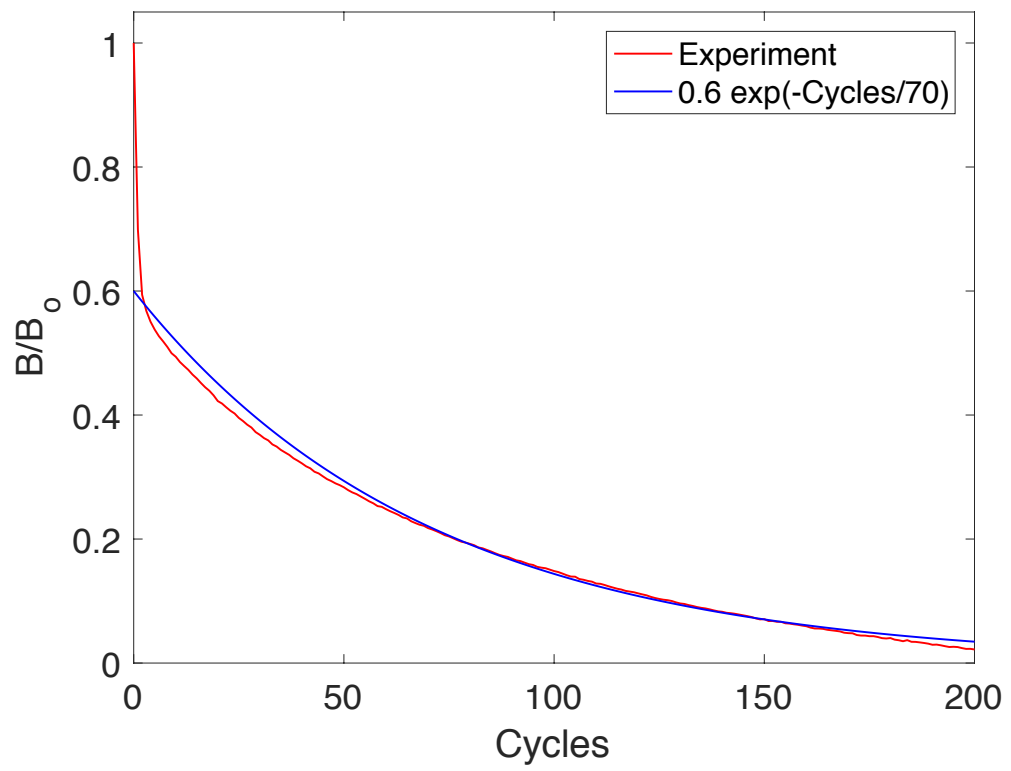

Fig.17a. The decay of the peak field a single tape

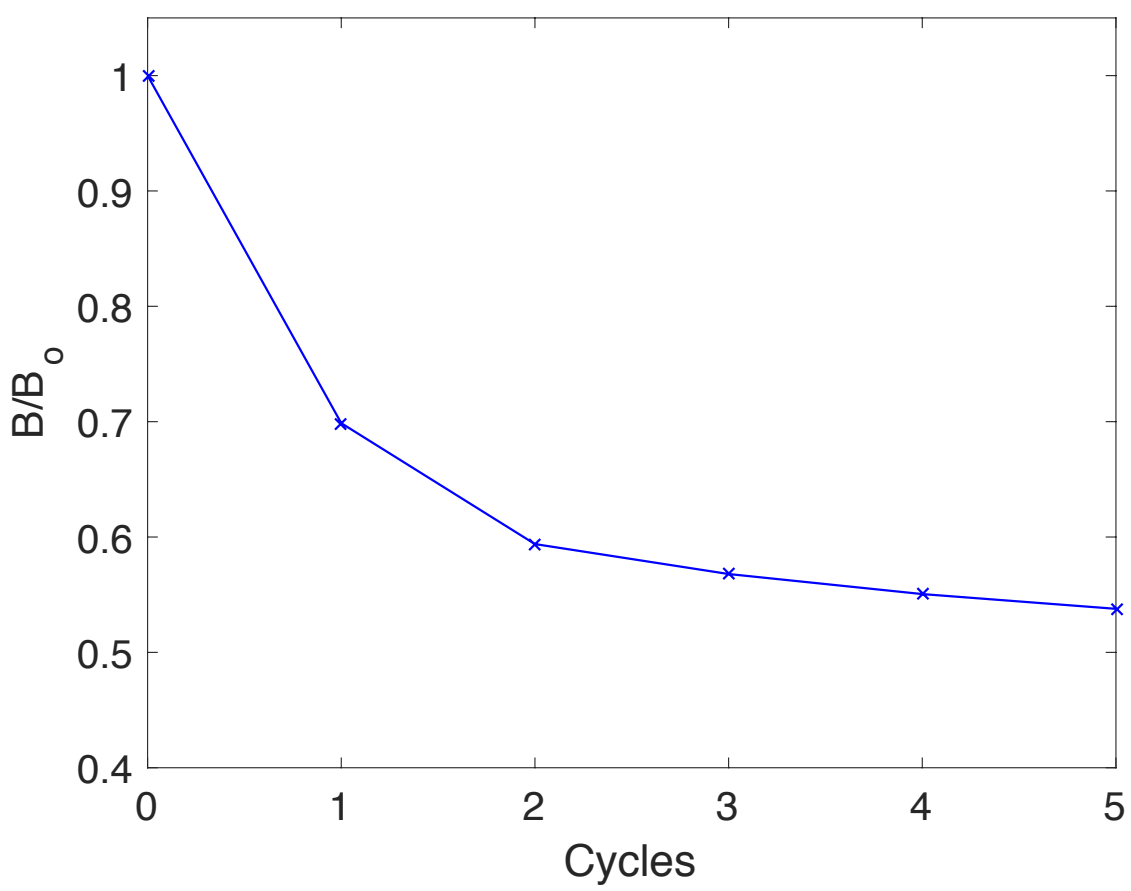

17b. The first few cycles of fig. 17 
Figs $17 \mathrm{a}$ and $17 \mathrm{~b}$ show the experimental ratio of the trapped central field to the starting value in a single tape in a crossed field of $120 \mathrm{mT}$. The sample was a $12 \mathrm{~mm} \times 12 \mathrm{~mm}$ square of Superpower tape with an $I_{c}$ of $300 \mathrm{~A}$. If $j_{c}$ is independent of $B$ this implies a penetration field $B_{p}=15.7 \mathrm{mT}$ and a trapped central field of $134 \mathrm{mT}$. The experimentally observed trapped field was $30 \mathrm{mT}$. There are two possible reasons for this discrepancy. One is that in a transport measurement the field is mostly around $B_{p}$ but the trapped field for constant $j_{c}$ is 20 times higher. This field will reduce $j_{c}$ significantly and lead to a lower trapped field than expected. The second reason is that in a transport measurement the field is parallel to the $a b$ planes, but the trapped field is normal to them, so the $j_{c}$ is lower due to its anisotropy.

If we assume a total layer thickness of 1 micron the $j_{c}$ is $2.5 \mathrm{MA} / \mathrm{cm}^{2}$. The theory predicts a decay constant of 225 cycles. Figure 17 shows a reasonable trapped flux up to about 150 cycles confirming that the thin YBCO layer causes a longer decay time. However there is an unexpected rapid drop in field in the first few cycles, followed by an exponential decay with a constant of 70 cycles. It seems likely this two step process is also caused by the anisotropy of $j_{c}$. Initially the field is parallel to the $c$ direction so $j_{c}$ is low, but as soon as the crossed field is applied the field direction is mainly parallel to the tapes so $\mathrm{j}_{\mathrm{c}}$ increases and the decay is slower. The variation of $j_{c}$ with $B$ could also have an effect, but it is likely to be lower.

\subsection{Stacked layers}

This work was prompted initially by the very slow decay time of a stack of tapes reported in ref (6), about 1000 cycles for a stack of sixteen tapes. Although the stacked tapes shield the interior ones from a perpendicular field, a parallel field can penetrate between them, so that to a first approximation they behave as single tapes. The Brandt-Mikitic theory does not cover multiple decoupled tapes, which will apply an inhomogeneous and changing field to each tape. A simulation of stacked layers using COMSOL and the $H$ formulation. is shown in fig. 18. The dimensions of the tape were $20 \mathrm{~mm}$ wide and $1 \mathrm{~mm}$ thick. It can be seen that the layers are behaving qualitatively as independent tapes.

\begin{tabular}{|c|c|c|c|c|}
\hline \multirow{2}{*}{ No. of Cycle } & \multicolumn{4}{|c|}{ Current Distribution (J) } \\
\hline & 1 Layer & 2 Layers & 4 Layers & 8 Layers \\
\hline 0 & & & & \\
\hline 1 & & & & \\
\hline 2 & & & & \\
\hline 3 & & & & \\
\hline 4 & & & & \\
\hline 5 & & & & \\
\hline 6 & & & & \\
\hline 7 & & & & \\
\hline 8 & & & & \\
\hline 9 & & & & \\
\hline 10 & & & & \\
\hline & & & & \\
\hline
\end{tabular}

Fig 18 The simulated current distribution in stacks of tapes 
Figure 19 shows the decay of trapped field for the two extremes, one tape and eight tapes. Here the average of the trapped field across the tape was measured, rather than the peak central field. It can be seen that the decay of the stack of eight tapes is significantly lower.

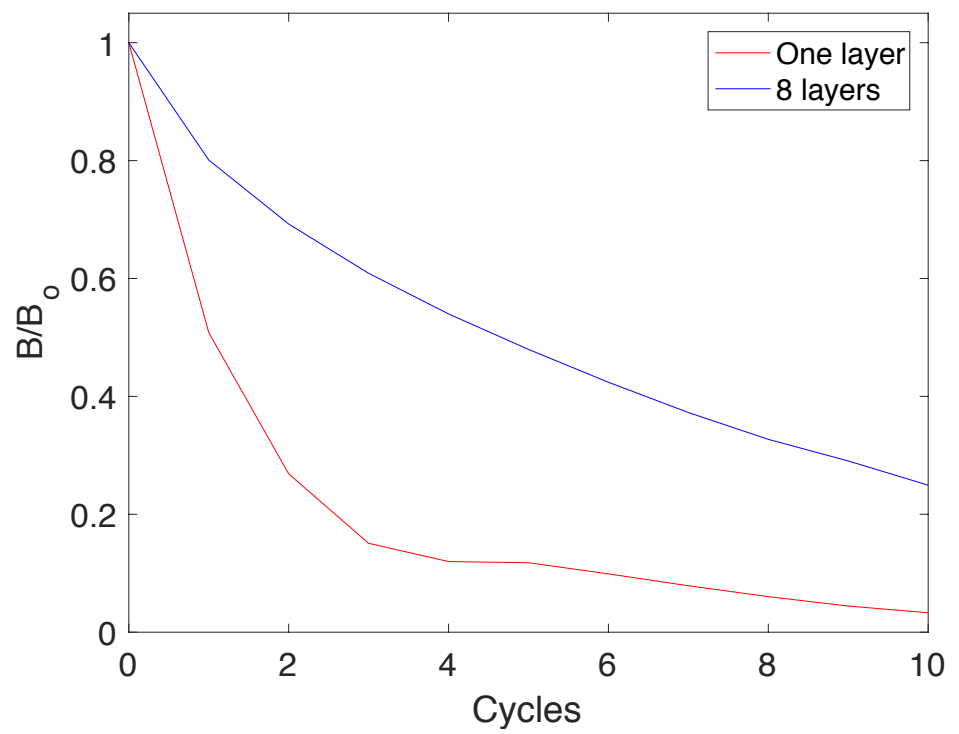

Fig.19. The simulated decay for one layer and eight layers.

Figure 20 shows how the decay constant varies with the number of tapes. It can be seen that the relationship is roughly linear.

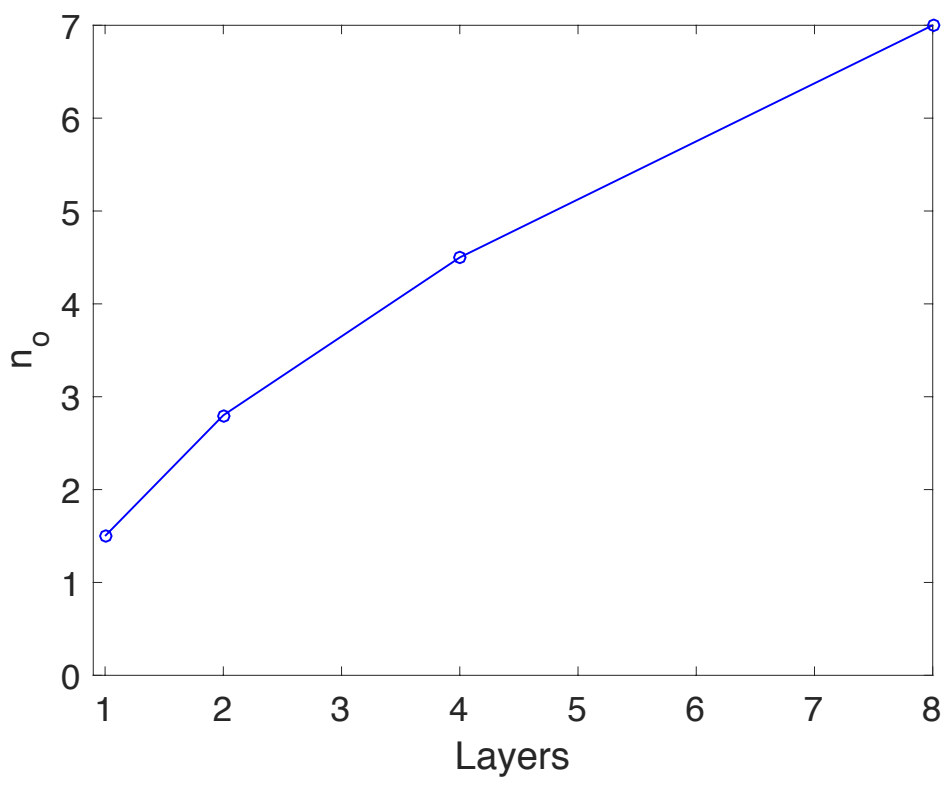

Fig.20 The decay constant as a function of the number of tapes. 
If we suppose the same holds for the YBCO tapes with a 1 micron layer for which the theory predicts a decay constant of 225 cycles the stack of sixteen would have a decay constant of 3600 which is actually larger than observed. However with many layers the field stays parallel to $c$ for much longer so the effective $j_{c}$ will be lower and the decay faster.

\section{Conclusions}

Experiments on the decay of trapped fields in stacks of YBCO tapes exposed to a transverse oscillating field had shown a remarkably slow decay constant even when the transverse field is much larger than the transverse penetration field $(6,7)$. Computer simulations based on the critical state, show good agreement between the Brandt-Mikitik theory and the decay of the magnetisation in long thin superconductors. These worked well for an aspect ratio of 20:1 in the regime where the transverse field was larger than the penetration field and the magnetisation decayed exponentially to zero. However it was not possible to simulate the very large aspect ratio of real YBCO tapes. When the transverse field was smaller than the penetration field the simulated magnetisation decreased as expected to the predicted value. However after many cycles the magnetisation did not stay constant but continued to decrease slowly. In very thin layers the movement of vortices is much smaller than in bulk and the effect of the reversible region was investigated. While movement is in the reversible range there is no critical state built up and so no decay. This can occur with very thin sheets. On the other hand there is a general principle that minor hysteresis loops do not close in the critical state so after many cycles the critical state must decay. Whether these effects are large enough to be observed remains to be seen, but they are confirmed in principle by simulations.

The simulations confirm the theoretical prediction that the decay in very thin sheets is slow, even if the applied transverse field is much greater than the transverse penetration field. They also show that the decay constant increases roughly in proportion to the number of tapes in a stack. These results can explain qualitatively the very slow decay of the magnetisation in refs $(6,7)$.

The experimental situation is more complex. The measured decay constants are qualitatively in agreement with both simulations and theory. In a bulk with $B_{p}>B_{T}$ the simulation shows a rapid drop over two cycles to the saturated value, followed by a slow further decrease. The theory predicts a similar rapid drop to the saturated magnetisation but no further decay. The experiments show a continuous decay over about 30 cycles. On the other hand for a single YBCO tape with $B_{p}<B_{T}$ the theory predicts a smooth decay with a constant of 200 cycles. However the experiments show a sharp drop in the first few cycles followed by a decay over 70 cycles. This discrepancy may be due to the anisotropy of $j_{c}$ and the change in angle of the field.

\section{References}

1) Sychev, V.V., Zenkeviteh, V.B., Andtianov, V.V., Baev, V.P., 'Influence of an Alternating Magnetic Field on Current Flowing through a Superconductor of Second Kind', Sov. Phys. JETP Lett., 9, 31-37, (1969)

2). N. Sakamoto, F. Irie, and K. Yamafuji, 'Flux Flow Voltage in a Type II Superconducting Foil in the Presence of a Superposed Perpendicular AC Field'., J. Phys. Soc. Jpn. 41, 32 (1976).

3). T. Ogasawara, Y. Takahashi, K. Kanbara, Y. Kubota, K. Yasohama and

K. Yasukochi, 'Alternating Field Losses in Superconducting Wires carrying DC Transport Currents: Part I Single Core Conductors', Cryogenics, 19, 736-740, (1979)

4) E. H. Brandt and G. P. Mikitik, 'Why an AC Magnetic Field Shifts the Irreversibility Line in TypeII Superconductors', Phys. Rev. Lett., 89, 027002-1 -027002-4, (2002). 
5) Ph. Vanderbemden, Z. Hong, T. A. Coombs, S. Denis, M. Ausloos, J. Schwartz, I. B. Rutel, N. Hari Babu, D. A. Cardwell, and A. M. Campbell, 'Behavior of Bulk High-temperature Superconductors of Finite Thickness Subjected to Crossed Magnetic Fields: Experiment and Model', Phys. Rev.B, 75, 174515 (2007)

6) M. Baghdadi, H. S. Ruiz and T. A. Coombs, 'Crossed-magnetic-field Experiments on Stacked Second Generation Superconducting Tapes: Reduction of the demagnetization effects', Appl. Phys. Lett. 104, 232602 (2014); http://dx.doi.org/10.1063/1.4879263

7) M. Baghdadi, H. S. Ruiz, J. F. Fagnard, M. Zhang, W. Wang, and T. A. Coombs, 'Investigation of Demagnetization in HTS Stacked Tapes Implemented in Electric Machines as a Result of Crossed Magnetic Field', IEEE Trans on Appl.Superconductivity, . 25, 6602404(2015)

8) S. Celebi, F. Sirois and C. Lacroix, 'Collapse of the Magnetization by the Application of Crossed Magnetic Fields: Observations in a Commercial Bi:2223/Ag tape and Comparison with Numerical Computations', Supercond. Sci. Technol. 28025012 (9pp), (2015) doi:10.1088/0953-2048/28/2/025012

9) A. M. Campbell, 'A new method of determining the critical state in superconductors', Superconductor Science \& Technology, vol. 20, pp. 292-295, 2007.

10) R. A. Doyle, A. M. Campbell and R. E. Somekh, 'Direct Observation of Intrinsic Pinning in YBCO Thin Films', Phys.Rev. Lett. volume 71, 4241-4244, (1993) 